\title{
A genuine four-partite entangled state
}

\author{
Ming-Yong Ye* and Xiu-Min Lin \\ School of Physics and Optoelectronics Technology, \\ Fujian Normal University, Fuzhou 350007, People's Republic of China
}

\begin{abstract}
In a recent paper, a genuine four-partite entangled state is proposed [Y. Yeo and W. K. Chua, Phys. Rev. Lett. 96, 060502 (2006)], which has been found to have many interesting entanglement properties. We show this state is locally equivalent to some graph states.
\end{abstract}

PACS numbers: 03.67.Hk, 03.67.Mn

Keywords: Entangled state; Graph state

*Electronic address: myye@mail.ustc.edu.cn 
Quantum entanglement has many applications such as teleportation [1] and dense coding [2]. Recently Yeo and Chua found a genuine four-qubit entangled state $\left|\chi^{00}\right\rangle$ that can be used to teleport an arbitrary two-qubit state [3]. The state $\left|\chi^{00}\right\rangle$ proposed in Ref. [3] has the form

$$
\left|\chi^{00}\right\rangle=\frac{1}{\sqrt{2}}\left(\left|\zeta^{0}\right\rangle+\left|\zeta^{1}\right\rangle\right)_{A_{3} A_{4} B_{1} B_{2}}
$$

with

$$
\begin{aligned}
& \left|\zeta^{0}\right\rangle=\frac{1}{2}(|0000\rangle-|0011\rangle-|0101\rangle+|0110\rangle), \\
& \left|\zeta^{1}\right\rangle=\frac{1}{2}(|1001\rangle+|1010\rangle+|1100\rangle+|1111\rangle) .
\end{aligned}
$$

It has been found that $\left|\chi^{00}\right\rangle$ has many interesting entanglement properties [3]. It has maximal entanglement between $A_{3} A_{4}$ and $B_{1} B_{2}$ or between $A_{3} B_{1}$ and $A_{4} B_{2}$, while it is not reducible to a pair of Bell states. The amount of entanglement between $A_{3} B_{2}$ and $A_{4} B_{1}$ has the value 1 when we use von Neumann entropy as an entanglement measure. It has properties that differ from those of four-party GHZ and W states [3]. Due to its interesting entanglement properties, the state $\left|\chi^{00}\right\rangle$ attracts much attention [4, 5, 6].

It is not clear why the state $\left|\chi^{00}\right\rangle$ has so many interesting entanglement properties. We will show $\left|\chi^{00}\right\rangle$ is locally equivalent to some graph states. First let us give a simple introduction to graph states [7]. Let $G=(V, E)$ be a graph, in which $V$ is the set of vertices and $E$ is the set of edges. The graph state $|G\rangle$ corresponding to the graph $G$ is the pure state

$$
|G\rangle=\prod_{\{a, b\} \in E} U_{a b}|+\rangle^{V}
$$

where $|+\rangle=(|0\rangle+|1\rangle) / \sqrt{2}$ and $U_{a b}$ is the controlled-phase gate

$$
U_{a b}=|0\rangle_{a}\left\langle\left. 0\right|_{a} \otimes I^{b}+\mid 1\right\rangle_{a}\left\langle\left. 1\right|_{a} \otimes \sigma_{z}^{b}\right.
$$

For an instance, the graph $G_{a}$ in FIG. 1(a) corresponds to the graph state

$$
\left|G_{a}\right\rangle=U_{A_{3} A_{4}} U_{B_{1} B_{2}} U_{A_{3} B_{1}} U_{A_{4} B_{2}}|+\rangle_{A_{3}}|+\rangle_{A_{4}}|+\rangle_{B_{1}}|+\rangle_{B_{2}}
$$

Two graphs $G=(V, E)$ and $G^{\prime}=\left(V, E^{\prime}\right)$ are locally equivalent, if there is a local unitary $U \in U(2)^{V}$ such that their corresponding graph states

$$
\left|G^{\prime}\right\rangle=U|G\rangle
$$


Local equivalence of graphs is connected to a graph transformation called local complementation: suppose $G=(V, E)$ is a graph and $a \in V$, the local complement of $G$ at $a$, denoted by $\tau_{a}(G)$, is a new graph obtained by complementing the neighborhood of $a$ and leaving the rest of the graph unchanged. It has been proved that two graphs $G=(V, E)$ and $G^{\prime}=\left(V, E^{\prime}\right)$ will be locally equivalent if they are related by a sequence of local complementations, i.e., $G^{\prime}=\tau_{a_{1}} \circ \ldots \circ \tau_{a_{n}}(G)$ for some $a_{1}, \ldots, a_{n} \in V$ [7, 8]. It can be easily found that the graph $G_{a}$ in FIG. 1(a) and the graph $G_{b}$ in FIG. 1(b) are locally equivalent because they are connected by a local complementation:

$$
G_{b}=\tau_{A_{4}}\left(G_{a}\right)
$$

The local equivalence between $G_{a}$ and $G_{b}$ can be confirmed by the relation between their corresponding graph states:

$$
\left|G_{b}\right\rangle=e^{i \frac{\pi}{4}} e^{-i \frac{\pi}{4} \sigma_{z}^{A_{3}}} e^{i \frac{\pi}{4} \sigma_{x}^{A_{4}}} e^{-i \frac{\pi}{4} \sigma_{z}^{B_{2}}}\left|G_{a}\right\rangle .
$$

Actually all graphs in FIG. 1 are locally equivalent since they are connected by local complementations. For more about graph states we refer to Ref. [7] and the references therein.

Our goal is to show $\left|\chi^{00}\right\rangle$ is locally equivalent to graph states depicted by graphs in FIG. 1. It can be easily checked that $\left|\chi^{00}\right\rangle$ is locally equivalent to $\left|G_{b}\right\rangle$ :

$$
\left|\chi^{00}\right\rangle=\sigma_{z}^{A_{3}} \sigma_{z}^{B_{2}} H^{B_{2}}\left|G_{b}\right\rangle
$$

where $H^{B_{2}}$ is the Hadamard gate acting on qubit $B_{2}$. Because graph states depicted in FIG. 1 are locally equivalent, the state $\left|\chi^{00}\right\rangle$ is locally equivalent to all these graph states. Since locally equivalent states have the same entanglement properties, the entanglement properties of $\left|\chi^{00}\right\rangle$ can be obtained by studying the graph state $\left|G_{a}\right\rangle$ or other locally equivalent graph states depicted in FIG. 1. It is not hard to find that $\left|G_{a}\right\rangle$ has all the above mentioned properties of $\left|\chi^{00}\right\rangle$.

Now we give two more properties about $\left|\chi^{00}\right\rangle$. It is known that graph states are also stabilizer states [7], and the graph state $\left|G_{b}\right\rangle$ is stabilized by the following four independent 
commuting observables:

$$
\begin{aligned}
& K_{1}=\sigma_{x}^{A_{3}} \sigma_{z}^{A_{4}} \sigma_{z}^{B_{1}} \sigma_{z}^{B_{2}}, \\
& K_{2}=\sigma_{z}^{A_{3}} \sigma_{x}^{A_{4}} \sigma_{z}^{B_{2}}, \\
& K_{3}=\sigma_{z}^{A_{3}} \sigma_{x}^{B_{1}} \sigma_{z}^{B_{2}} \\
& K_{4}=\sigma_{z}^{A_{3}} \sigma_{z}^{A_{4}} \sigma_{z}^{B_{1}} \sigma_{x}^{B_{2}} .
\end{aligned}
$$

From Eq. (10) we know $\left|\chi^{00}\right\rangle$ is also a stabilizer state and it is stabilized by

$$
\begin{aligned}
& \bar{K}_{1}=\sigma_{z}^{A_{3}} \sigma_{z}^{B_{2}} H^{B_{2}} K_{1} \sigma_{z}^{A_{3}} H^{B_{2}} \sigma_{z}^{B_{2}}=\sigma_{x}^{A_{3}} \sigma_{z}^{A_{4}} \sigma_{z}^{B_{1}} \sigma_{x}^{B_{2}}, \\
& \bar{K}_{2}=\sigma_{z}^{A_{3}} \sigma_{z}^{B_{2}} H^{B_{2}} K_{2} \sigma_{z}^{A_{3}} H^{B_{2}} \sigma_{z}^{B_{2}}=-\sigma_{z}^{A_{3}} \sigma_{x}^{A_{4}} \sigma_{x}^{B_{2}}, \\
& \bar{K}_{3}=\sigma_{z}^{A_{3}} \sigma_{z}^{B_{2}} H^{B_{2}} K_{3} \sigma_{z}^{A_{3}} H^{B_{2}} \sigma_{z}^{B_{2}}=-\sigma_{z}^{A_{3}} \sigma_{x}^{B_{1}} \sigma_{x}^{B_{2}}, \\
& \bar{K}_{4}=\sigma_{z}^{A_{3}} \sigma_{z}^{B_{2}} H^{B_{2}} K_{4} \sigma_{z}^{A_{3}} H^{B_{2}} \sigma_{z}^{B_{2}}=\sigma_{z}^{A_{3}} \sigma_{z}^{A_{4}} \sigma_{z}^{B_{1}} \sigma_{z}^{B_{2}},
\end{aligned}
$$

that is to say

$$
\bar{K}_{i}\left|\chi^{00}\right\rangle=\left|\chi^{00}\right\rangle, i=1, \ldots, 4 .
$$

Eq. (19) can be directly checked from the expressions of $\left|\chi^{00}\right\rangle$ and $\bar{K}_{i}$.

Another property about $\left|\chi^{00}\right\rangle$ is that it also admits a GHZ-argument [9]. According to observables $\bar{K}_{1}, \bar{K}_{2}, \bar{K}_{4}$ and

$$
\bar{K}_{1} \bar{K}_{2} \bar{K}_{4}=\sigma_{x}^{A_{3}} \sigma_{x}^{A_{4}} \sigma_{z}^{B_{2}}
$$

we can design four different measurement settings. In each measurement setting, the measurement results on different qubits will have a correlation. The observables $\bar{K}_{1}, \bar{K}_{2}, \bar{K}_{4}$ and $\bar{K}_{1} \bar{K}_{2} \bar{K}_{4}$ lead to the following correlations respectively

$$
\begin{aligned}
m_{x}^{A_{3}} m_{z}^{A_{4}} m_{z}^{B_{1}} m_{x}^{B_{2}} & =1, \\
-m_{z}^{A_{3}} m_{x}^{A_{4}} m_{x}^{B_{2}} & =1, \\
m_{z}^{A_{3}} m_{z}^{A_{4}} m_{z}^{B_{1}} m_{z}^{B_{2}} & =1, \\
m_{x}^{A_{3}} m_{x}^{A_{4}} m_{z}^{B_{2}} & =1,
\end{aligned}
$$

where $m_{x}^{A_{3}}= \pm 1$ and $m_{z}^{A_{3}}= \pm 1$ denote measurement outcomes if the qubit $A_{3}$ is measured in $\operatorname{spin}-x$ or spin- $z$ direction. In local hidden variable theories all these four Eqs. (21,24) should be satisfied at the same time. However the product of Eq. (21), (22) and (23) gives

$$
m_{x}^{A_{3}} m_{x}^{A_{4}} m_{z}^{B_{2}}=-1
$$


which contradict with Eq. (24), so local hidden variable theories cannot be used to explain the measurement results on state $\left|\chi^{00}\right\rangle$.

In conclusion we have shown that the interesting four-partite genuine entangled state $\left|\chi^{00}\right\rangle$ proposed in [3] is locally equivalent to graph states depicted by graphs in FIG. 1. This finding uncovers the mysteries of the state $\left|\chi^{00}\right\rangle$ and gives an explanation why it has so many interesting entanglement properties. We have also shown $\left|\chi^{00}\right\rangle$ is a stabilizer state and there is a GHZ-argument for $\left|\chi^{00}\right\rangle$.

This work was funded by National Natural Science Foundation of China (Grant No. 10574022), the Fujian Provincial Natural Science Foundation (Grant No. A0410016 and No. 2006J0230), and the Foundation for Universities in Fujian Province (Grant No. 2007F5041).

[1] C. H. Bennett, G. Brassard, C. Crepeau, R. Jozsa, A. Peres, and W. K. Wootters, Phys. Rev. Lett. 70 (1993) 1895.

[2] C. H. Bennett and S. J. Wiesner, Phys. Rev. Lett. 69 (1992) 2881.

[3] Y. Yeo and W. K. Chua, Phys. Rev. Lett. 96 (2006) 060502.

[4] C. Wu, Y. Yeo, L. C. Kwek, and C. H. Oh, Phys. Rev. A 75 (2007) 032332.

[5] P. X. Chen, S. Y. Zhu, and G. C. Guo, Phys. Rev. A 74 (2006) 032324.

[6] Z. X. Man, Y. J. Xia, and N. B. An, Phys. Rev. A 75 (2007) 052306.

[7] M. Hein, W. Dür, J. Eisert, R. Raussendorf, M. V. D. Nest, and H. J. Briegel, quant-ph/0602096 v1.

[8] M. Van den Nest, J. Dehaene, and B. De Moor, Phys. Rev. A 69 (2004) 022316.

[9] D. M. Greenberger, M. Horne, and A. Zeilinger, Bell's Theorem, Quantum Theory and Conception of the Universe, edited by M. Kafatos et al., (Kluwer, Dordrecht, 1989), p. 69. 


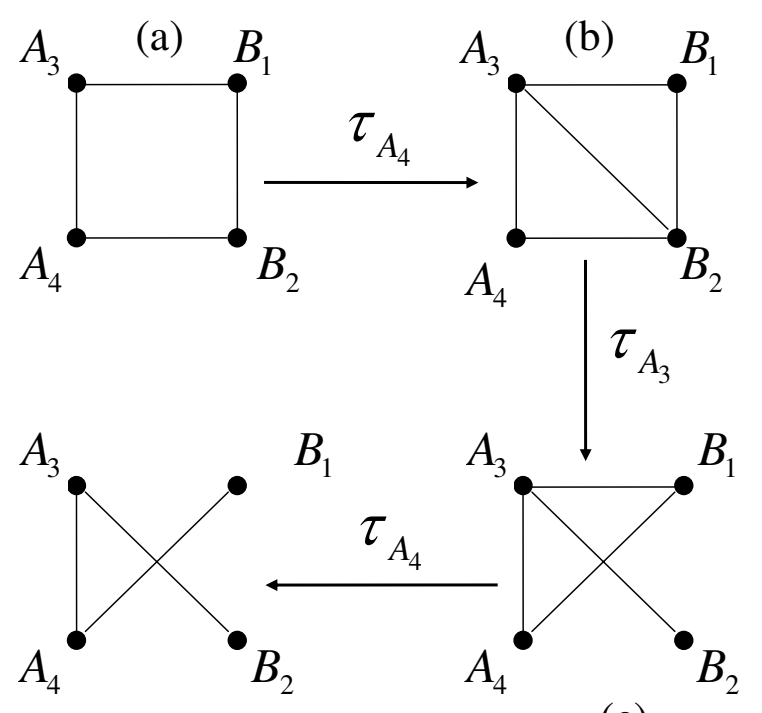

(d)

(c)

FIG. 1: Four graphs are connected by local complementations: $G_{b}=\tau_{A_{4}}\left(G_{a}\right), G_{c}=\tau_{A_{3}}\left(G_{b}\right), G_{d}=$ $\tau_{A_{4}}\left(G_{c}\right)$. Their corresponding graph states $\left|G_{i}\right\rangle, i=a, b, c, d$, are locally equivalent, and they are locally equivalent to $\left|\chi^{00}\right\rangle$. 